\title{
Concerns, challenges and directions of development for the issue of representing uncertainty in risk assessment
}

Roger Flage ${ }^{1, *}$, Terje Aven $^{1}$, Enrico $\mathrm{Zio}^{2,3}$ \& Piero Baraldi ${ }^{3}$

${ }^{1}$ University of Stavanger, Norway

${ }^{2}$ Chair on Systems Science and the Energetic Challenge, European Foundation for New Energy-

EDF, Ecole Centrale Paris and Supelec, France

${ }^{3}$ Energy Department, Politecnico di Milan, Italy

* Please address correspondence to Roger Flage, Department of industrial economics, risk management and planning, Faculty of Science and Technology, University of Stavanger, 4036 Stavanger, Norway. E-mail: roger.flage@uis.no. 


\begin{abstract}
In the analysis of the risk associated to rare events that may lead to catastrophic consequences with large uncertainty, it is questionable that the knowledge and information available for the analysis can be reflected properly by probabilities. Approaches other than purely probabilistic have been suggested, for example using interval probabilities, possibilistic measures, or qualitative methods. In the present paper, we look into the problem and identify a number of issues which are foundational for its treatment. The foundational issues addressed reflect on the position that "probability is perfect" and take into open consideration the need for an extended framework for risk assessment that reflects the separation that practically exists between analyst and decision maker.
\end{abstract}

Key words: risk assessment, epistemic uncertainty, representation, decision-making 


\section{INTRODUCTION}

Probabilistic analysis has now been used for more than 30 years (ref. the Reactor Safety Study, WASH-1400 [1]) as the basis for the analytical and quantitative process of risk assessment; see the reviews by Rechard [2,3]. A probabilistic risk assessment (PRA), sometimes also referred to as a quantitative risk assessment (QRA), systemises the knowledge, information and uncertainties about the phenomena involved in the activities studied: what are the possible hazards and threats, their causes and consequences? What are the probabilities of occurrence of the accident scenarios leading to these consequences? Knowledge is input into the QRA model and the associated uncertainties are expressed with probabilistic metrics; then, also the risk metrics in output are represented in terms of probabilistic metrics, to display the uncertainties; see e.g. the review by Jonkman et al. [4].

In a practical QRA setting it is common to distinguish between aleatory (stochastic) and epistemic (knowledge-related) uncertainty [5,6,7]. The former refers to variation in large populations, is typically measured using frequentist probabilities and is considered irreducible; the latter refers to lack of knowledge about phenomena (usually translating into uncertainty about the parameters of a model used to describe some phenomenon or phenomena), is typically measured using subjective probability and is considered reducible. Winkler [8] describes the distinction between types of uncertainty - aleatory and epistemic uncertainty in particular - as fundamentally flawed but, nevertheless (and in line with Apostolakis [9]), as useful for the practice of modelling and analysing complex systems. Apostolakis states [9 p. 1362]: 
'There is only one kind of uncertainty stemming from our lack of knowledge concerning the truth of a proposition [...] [Distinctions between probabilities are] merely for our convenience in investigating complex phenomena. Probability is always a measure of degree of belief.'

As for the objective/subjective distinction that is often tacitly taken to underlie the frequentist/subjective probability distinction, Winkler writes [8 p. 128]:

\footnotetext{
'To some individuals, especially those trained in a scientific environment, the subjectivity in subjective probability seems to be at odds with a scientific search for objectivity. But, in fact, objectivity in dealing with uncertainty in the real world is a goal that is elusive at best and might more realistically be viewed as unattainable. In order to apply the classical or relative-frequency interpretations of probability, certain assumptions must be made, and these are ultimately subjective assumptions (Gelman et al. $\left.{ }^{3}\right)$.'
}

As for measurement, Popper distinguishes three worlds (see e.g. [10]): the physical world, the personal and subjective world, and the objective world of others; in addition, at the intersection of the second and third worlds, is the shared inter-subjective world. Measurement of properties of the physical world, such as the length of an object, is different from measurement of uncertainty in the sense of lack of knowledge, expressed by subjective probability reflecting a person's degree of belief. Some (e.g. [10,11]) would refer to this as measurement of hard and soft systems, respectively.

Probabilistic analysis is the predominant method used to handle the uncertainties involved in risk analysis, both of aleatory and epistemic types. It is also used in the quantification of margins and uncertainties in analyses that use computational models to predict complex system behaviour [12]. It seems correct to say that probability is indeed perfectly suited to describe aleatory 
uncertainty, in its limiting relative frequency interpretation. When used as representation of epistemic uncertainty, the suitable interpretation is the subjective one typical of the Bayesian probabilistic framework $[13,14]$. By subjective probability is here meant the exact assignment, say, $\mathrm{P}(\mathrm{A})=0.3$, thus "à la Savage" [15]. Of course, if we judge the event A to be part of an exchangeable sequence we would introduce a chance/propensity $\mathrm{p}$ of $\mathrm{A}$, understood as the limiting relative frequency of $\mathrm{A}$ in an infinite exchangeable sequence; then proceed to assign $\mathrm{P}(\mathrm{A} \mid \mathrm{p})=\mathrm{p}$ and establish a (prior) probability distribution $\mathrm{F}\left(\mathrm{p}^{\prime}\right)=\mathrm{P}\left(\mathrm{p} \leq \mathrm{p}^{\prime}\right)$ on $\mathrm{p}$. Here $\mathrm{P}(\mathrm{A})$ and $\mathrm{F}\left(\mathrm{p}^{\prime}\right)$ are subjective probabilities expressing degrees of beliefs, and both $\mathrm{P}(\mathrm{A})$, determined as the integral of $\mathrm{P}(\mathrm{A} \mid \mathrm{p})$ with respect to $\mathrm{F}$, as well as the value of $\mathrm{F}\left(\mathrm{p}^{\prime}\right)$ for a given $\mathrm{p}^{\prime}$, are fixed, which is the point we are making above.

However, many researchers have to greater or lesser extent criticised probability as a representation of epistemic uncertainty. A number of alternative representations of epistemic uncertainty have been proposed, including imprecise/interval probability [16,17,18,19,20], probability bounds (p-box) analysis [21,22], fuzzy set theory [23,24], fuzzy probability [25], the theory of possibility $[26,27,28]$ and the theory of belief functions (evidence theory/DempsterShafer theory) [29]. Some amend the aleatory and epistemic uncertainty concepts by concepts such as fuzziness, ambiguity and vagueness, to be handled using fuzzy set theory; see e.g. [30,31]. Other (e.g. [13]) argue against this, saying for example that uncertainty must be distinguished from ambiguity, which must be removed before it is meaningful to discuss uncertainty (removed here means reduced to a desirable or practicable level, which is assumed to always be possible). In the present paper, we do not look into fuzzy set theory and related representations, as their motivation is not to describe epistemic uncertainty about unknown true quantities but to provide uncertainty statements about vague or ambiguous statements such as 
"few leakages". Formally this means that we restrict attention to classical (binary) set theory. See also the special issues of JRR [32], JSTP [33] and RESS [34] on the subject of alternative representations of uncertainty. One way to categorise alternative representations is [35,36]:

a) Probability-bound analysis, combining probability analysis and interval analysis [37]. Interval analysis is used for those components whose aleatory uncertainties cannot be accurately estimated; for the other components, traditional probabilistic analysis is carried out.

b) Imprecise probability, after Walley [19] and the robust statistics area [38] (see also $[39,40])$, which encompasses probability-bound analysis, and certain aspects of evidence and possibility theory as special cases.

c) Evidence theory (or belief function theory) as proposed by Dempster [41] and Shafer [29], and the closely linked theory of random sets [42].

d) Possibility theory $[26,27,43]$, which is formally a special case of the imprecise probability and random set theories (see Destercke et al. [53] for some theoretical links between random sets, possibility functions, probability intervals, as well as so-called p-boxes and clouds).

For a unified overview of various representations of uncertainty that have arisen in the areas of artificial intelligence and decision theory during the last fifty years, reference is made to [43].

The above mentioned methods produce epistemic-based uncertainty descriptions and in particular intervals. They have not been broadly accepted in the risk assessment community. Although much effort has been made in the area of alternative uncertainty representations there are still 
many open questions related to the foundations of these approaches and their use in both inference as well as risk and uncertainty decision-making; see for example the discussions in [44, 45, 46, 47, 48, 49, 50, 51]. Many risk researchers and risk analysts are skeptical towards the use of the alternative approaches (such as those of the four categories a) - d) mentioned above) for the representation and treatment of uncertainty in risk assessment for decision-making, and some also argue intensively against them; see, for example [50 p. 280]. According to Cooke [52] alternative representations have been on the decline for at least the last 20 or so years in the artificial intelligence community, having been introduced in relation to expert systems in the 1970's leading to 'an explosion of "alternative representations of uncertainty" through the 1980's' [52 p. 14]. So although re-emerging in new fields, as Cooke [52] also points out, the field where it was introduced apparently to an increasing and lately dominant extent prefers the Bayesian framework over alternative representation. One main objection raised, as we will come back to in Section 4, is lack of operational meanings, or interpretations, for these representations.

Most developments relating to the non-probabilistic and hybrid representations of uncertainty address technical issues, whereas less is said on foundational issues and little can be found on principles and guidelines for selection and use in the practice of risk assessment. In the present paper, we attempt to address that side of the problem and identify directions and needs for development. We discuss the needs for probability and the challenges in the use of nonprobabilistic representations of uncertainties. We address the practical issues coming from the fact that the risk assessment is conducted by an analyst and the results are used by one or more decision-makers, and the complications which arise from subjectivity and separation. Axiomatic differences between uncertainty representations is hence outside the scope of the present paper; we refer to [53] for a discussion on this topic. Furthermore, a critical review and discussion of 
evidence theory and possibility theory in the context of system analysis is offered in [54], focusing on inter alia formal properties and combination of knowledge.

The paper tries to get right to the point as follows: in Sections 2 through 6 we recall the differences in the alternative representations of uncertainties, and present and discuss the issues; in Section 3 we provide an overall discussion and in Section 8 we draw some conclusions and provide some ideas of directions for development.

\section{SUBJECTIVE PROBABILITY}

In the Bayesian probabilistic framework of representation of epistemic uncertainty, probability is interpreted as a subjective measure of uncertainty - an expression of degree of belief of the analyst, then governed by the axioms of probability. The term subjective probability is most commonly used, but we see also other words adopted such as judgmental probability and knowledge-based probability. By subjective probability here is meant an exact assignment, say $\mathrm{P}(\mathrm{A})=0.3$. Two interpretations are described below, one making reference to betting and the other to a standard for measurement of uncertainty.

If linked to betting, the probability of an event $\mathrm{A}, \mathrm{P}(\mathrm{A})$, equals the amount of money that the analyst(s) assigning the probability would be willing to bet if a single unit of payment would be

given in return in the case that the event A were to occur, and nothing otherwise. The opposite bet must also hold, i.e. the assessor must be willing to bet the amount $1-\mathrm{P}(\mathrm{A})$ if a single unit of 
payment would be given in return in the case that $\mathrm{A}$ were not to occur, and nothing otherwise $[56]$.

If linked to the standard for measurement of uncertainty, the probability $\mathrm{P}(\mathrm{A})$ is the number such that the uncertainty about the occurrence of event $\mathrm{A}$ is considered equivalent to the uncertainty about the occurrence of some standard event, e.g. drawing a red ball at random from an urn that contains $\mathrm{P}(\mathrm{A}) \times 100 \%$ red balls and $1-\mathrm{P}(\mathrm{A}) \times 100 \%$ balls of colors other than red (e.g. $[57,58,59,60])$.

Several authors argue that the problem of using probability in application lies with the measurement procedures and not with the probability concept in itself (e.g. $[59,60])$. One development direction then, as suggested by Lindley [59] and O'Hagan \& Oakley [61], is to retain probability as the sole representation of uncertainty and to focus on improving the measurement procedures for probability. The thesis is that no alternative to probability is needed, as "probability is perfect" $[58,61,62]$. These authors acknowledge that there is a problem of imprecision in probability assignments, but that is considered a problem related to the elicitation of probabilities, and not a problem of the probability concept.

This is a strong thesis that in our view is not justifiable as a general statement. We need to clarify the situations addressed, making some distinctions important for the practice of risk assessment and management. The classical case of decision analysis for risk management considers the situation in which the assessor of the probabilities and risk is also the decision maker: in this case, it is possible to argue that the use of (subjective) probability as the only uncertainty measure is proper, because the subjectivity of the assessment is brought in the decision scheme coherently 
by the assessor herself/himself. In simple words, the assessor expresses her/his judgements to arrive at the assessment of the probabilities which she/he uses for the decision making. However, the situation most commonly encountered in practice is different, wherein there is one (or more) assessor(s) who perform the (probabilistic) risk assessment whose results are fed to decision makers other than the assessor(s). What characterise such situations is specifically:

1. A risk assessment is carried out by a risk analyst/expert group following a request from the decision maker(s) (or another stakeholder(s)). The assessment is dependent on the subjective expertise of the assessor(s).

2. The aim is to carry out the assessment, whose results are independent of the decision maker (and other stakeholders).

3. Several stakeholders other than the decision maker will often be informed by the assessment and its results.

4. The decision maker(s) and stakeholders will perform the decision making process according to their subjective values and preferences, and be informed by the risk results performed by the assessor(s) on the basis of her/his (subjective) expertise.

An example is a societal safety issue, where politicians are to make decisions on protection measures, informed by the results of a risk assessment characterising the risks associated to alternative options, where the risk assessment is performed by expert risk analysts.

The issue of separation between analyst and decision-maker is of course a transversal one and hence relevant regardless of whether the uncertainty representation is subjective probability or another method. 
It is clear that in these situations, the decisions taken by the decision makers are influenced also by the knowledge that the assessors put in the risk assessment, through the output probabilities calculated. The depth of the knowledge about the system and phenomena studied determines the strength of the assessment, and of its influence on the decision maker. On the contrary, when knowledge is poor, this should be transparent and acknowledged in the decision process which relies on the provided probabilities.

In the above scenario of decision making, we advocate strongly that the output that should be retained from the risk assessment and accounted for in the decision making process comprises two main components:

a) The quantitative description given by the uncertainty measure used $Q$ (for example, probability $\mathrm{P})$.

b) The background knowledge $\mathrm{K}$ used in the assessment.

We regard this as fundamentally important, because the probabilities of the risk assessment are by definition subjective, or inter-subjective to some extent, depending on the situation analysed. The point we are trying to convey is that the subjective probability $\mathrm{P}(\mathrm{A})$ can be written as $\mathrm{P}(\mathrm{A} \mid \mathrm{K})$, where $\mathrm{K}$ denotes the background knowledge that the probability is based on. We can have $P\left(A \mid K_{1}\right)=P\left(A \mid K_{2}\right)$ for vastly different background knowledge bases $K_{1}$ and $K_{2}$, e.g. one (say $K_{1}$ ) includes reliable models, abundant relevant data, strong agreement among experts and, in particular, weak assumptions because of the strong knowledge on the system and the related events and phenomena, whereas the other $\left(\mathrm{K}_{2}\right)$ includes less reliable models, little or no relevant 
data, disagreement among experts and strong assumptions because of the weak knowledge on the system and the related events and phenomena. When performing a risk assessment, the background knowledge is to large extent expressed in the assumptions made. In case of relatively poor knowledge, stronger assumptions are made. With strong knowledge, lighter assumptions are required. Table I presents a matrix of different situations reflecting different "states" of Q and K.

Table I: A matrix reflecting different situations of uncertainty descriptions Q and knowledge $\mathrm{K}$ (the numbers are just references for these elements of the matrix).

\begin{tabular}{|l|l|l|l|}
\hline Uncertainty measure Q & Subjective & Inter-subjectivity & Broad inter- \\
Knowledge K & among experts & subjectivity \\
\hline Strong: & $(3)$ & $(5)$ \\
in the risk assessments & & $(2)$ & \\
\hline $\begin{array}{l}\text { Medium: } \\
\text { Moderately strong }\end{array}$ & & & \\
assumptions made in the & & & \\
risk assessments & & & \\
\hline Poor: & & & \\
Strong assumptions & & & \\
made in the risk & & & \\
\hline
\end{tabular}


Consider the case (1), which is characterised by strong assumptions and a subjective measure of uncertainty, understood as a subjective probability. By replacing the probability $\mathrm{Q}$ by an interval probability, the intention is to move the situation to (2), (3), (4) or (5). The idea is to use intervals that are less subjective and based on not so strong assumptions. In most cases the change would lead to the situation (2), as the intervals would need also to be based on some assumptions and there are always aspects of the uncertainty description that are not generally inter-subjective.

Arguably it may be no easier to obtain agreement on a lower and upper probability than on an exact probability. However, in some cases it could be possible to find intervals that experts could agree on. Suppose that we have agreement on the conditional probability $\mathrm{P}(\mathrm{A} \mid \mathrm{X})$, but disagreement on the probability distribution of $\mathrm{X}$, where $\mathrm{X}$ is known to take a value in the interval $\left[\mathrm{x}_{\min }, \mathrm{x}_{\max }\right]$. Then we may at least obtain agreement on the probability interval $\left[\mathrm{P}\left(\mathrm{A} \mid \mathrm{x}_{\min }\right)\right.$, $\left.\mathrm{P}\left(\mathrm{A} \mid \mathrm{x}_{\max }\right)\right]$ (assuming that $\mathrm{P}(\mathrm{A} \mid \mathrm{x})$ is increasing in $\mathrm{x}$ ), although of course this could lead to a very wide interval.

We stress that we perform no value ranking of the different elements of the matrix in Table 1. A subjective assignment (1) may well serve the purpose of the assessment in some cases, where focus is on reporting some analysts' view on specific issues. The results are acknowledged as subjective but still considered informative for the relevant decision making. In other cases, we may look for more inter-subjective results, more independent of the judgements of specific analysts or experts. Normally we find that a combination of different approaches can be useful to support the decision making. We need to produce both subjective judgments and beliefs by selected analysts and experts, and we need to produce more inter-subjective results where the 
knowledge and lack of knowledge available are laid out 'plain and flat' with no additional information inserted.

\section{LOWER AND UPPER PROBABILITIES}

Interval or imprecise probabilities are proposed as an uncertainty measure, alternative to "precise" (single-valued) subjective probabilities. The motivation is that intervals in many cases correspond better to the (weak) information available. The intervals can be elicited by direct arguments or constructed indirectly from assigned possibility functions or mass functions, in the framework of evidence theory.

Consider the problem of (subjectively) describing the uncertainty of a quantity $\mathrm{x}$ which is known to take value $1,2,3,4$ or 5 . The basis for the assignment of the probabilities of the different values is rather weak, because of little knowledge on the process generating x, i.e. because of large epistemic uncertainties. Using precise probabilities, the analyst is required to specify five values, i.e. one probability for each one of the possible values of $x, 1,2,3,4$ or 5 (so, actually, only four probabilities are needed as their sum must be 1). Based on the scarce knowledge of the process, the analyst might find it difficult to assign a specific, precise probability mass to each of the 5 possible values of $\mathrm{x}$ : a distribution like for example $0.1,0.2,0.3,0.1,0.3$ may leave the analyst with the uneasy feeling that the numbers are somewhat arbitrary given the rather weak background for the assignments. Yet the assessor may find it practically feasible as she/he has just four numbers to assign and feels that the values assigned indeed are reflecting her/his best judgment. Alternatively, she/he could opt for a uniform distribution to reflect that she/he has the 
same degree of belief in $\mathrm{x}$ to be equal to, say, 2 as in $\mathrm{x}$ to be equal to, say, 5 or any other value. On the premise that such a distribution does, in fact, reflect the assessor's uncertainties, it is attractive to use it as only one value is needed.

In principle, the assignment of intervals would seem more fit to a situation of scarce knowledge of the process, which leads to a lack of precision in the value assignment of probabilities. This is because the analyst need not specify one exact number; on the contrary, she/he is given a way to reflect her/his limited knowledge, and her/his associated uncertainty in the assignments, through an imprecise (interval) specification. However, the matter of fact is that in practice the analyst is still required to assign numbers, and now not just one for each $\mathrm{x}$ but two, a lower and an upper bound reflecting the imprecision of the assignment in view of the scarce knowledge. Take for example $x=3$. The question to address is: How likely is it that $x=3$ ? The analyst finds it difficult to specify just one number in $[0,1]$; but how, then, does she/he assign the bounds? One way is direct assignment, e.g. say, that the probability of $x=3$ lies in the interval $[0.2,0.5]$; but, again, one may feel that the assignment of such an interval is somewhat arbitrary and now, actually, we have a double assignment (lower and upper bounds of the intervals), with double source of arbitrariness. Also, it is challenging to really interpret what the assigned interval expresses. First of all, it expresses that the analyst is not willing to specify her/his degree of belief on the probability of $\mathrm{x}=3$ more precisely than $[0.2,0.5]$. It also states that the analyst's degree of belief on the realization of the value $\mathrm{x}=3$ is higher than that she/he has when drawing a specific ball out of an urn having 5 balls, but lower than when drawing a ball from an urn having 2 balls. She/he is not willing to specify her/his beliefs on the value of $\mathrm{x}$ further than this. This reasoning is not straightforward and it might be difficult to "absorb" by the assessors who are asked to provide the 
assignments. It is our experience that the assessors need a lot of training and practice to get used to this way of thinking.

In addition, many assessors struggle with understanding what is gained by the use of interval probability assignments compared to exact numbers. In the end, the richer description provided by the intervals, which are capable of representing the imprecision in the assignments, is contained in a double set of numbers, whose message is far more difficult to read than single point values. With the difficulties that they encounter in assigning the numbers, they question the need for going beyond one uncertainty description level, as provided by single-valued probabilities.

This reflection is important for the practice of risk assessment and the consequential decision making. To be able to effectively use interval probabilities in practice, the obstacles in the interval assignments need to be identified, addressed and adequately dealt with. More, and extensive, research needs to be carried out in the directions of interval elicitations and interval interpretations. Until solid solutions are offered, pragmatically, our recommendations for cases in which the background knowledge is poor and not given in a clear structured form, are:

- still use exact probabilities, but supplemented with an explicit characterization of the background knowledge, e.g. by a qualitative approach for assessing the importance of the assumptions that the quantitative analysis is based on (see Section 6).

- use interval probabilities to supplement the exact probabilities if the format of the information and knowledge available justifies the assignment of a specific interval of values, for example if a specific possibility function can be derived, or the expert 
judgments are elicited in a form that supports the use of intervals. For instance, suppose that the experts provide information about $\mathrm{x}$ of the form: there is nothing to suggest that the value of $\mathrm{x}$ is or is not 3 ; for the remaining $\mathrm{x}$ values $1,2,4$ and 5 , my upper probabilities are $0.2,0.5,0.3$ and 0.7 . These elicited expert judgments could be well reflected by the set of probability intervals $[0,0.2],[0,0.5],[0,1],[0,0.3]$ and $[0,0.7]$.

\section{4. "POSSIBILITY", "BELIEF", AND OTHER NON-PROBABILISTIC MEASURES OF UNCERTAINTY}

Both probability-bound and imprecise probability analyses can be seen as extensions of probability analysis. Their common, starting ground is that single-valued probability is not considered adequate for representing uncertainty and the solution called for is a representation of uncertainty based on measures interpreted as lower and upper probabilities. Also the representations based on evidence theory (belief and plausibility measures) and possibility theory (necessity and possibility measures) can be interpreted as lower and upper probabilities; in fact, technically both possibility theory and probability theory are special cases of evidence theory. However, belief measures and possibility measures can also be understood as expressing “degrees of belief" and "degrees of possibility" per se, and not as lower and upper probabilities. This is how belief functions are to be understood according to Shafer [29], who presents evidence theory as a generalisation of the Bayesian theory of subjective probability in the sense that it does not require probabilities for each proposition or event of interest but bases the belief in the truth of a proposition or occurrence of an event on the probabilities of other propositions or events related to it. Shafer [63] uses several metaphors for assigning (and hence interpreting) a belief 
function Bel. The simplest says that $\operatorname{Bel}(A)=q$ means that the assessor judges the strength of the evidence indicating that event $\mathrm{A}$ is true, is comparable with that of the evidence provided by a witness who has a q x $100 \%$ chance of being reliable, i.e.

$\operatorname{Bel}(\mathrm{A})=\mathrm{P}($ the witness claiming that $\mathrm{A}$ is true, is reliable $)$.

Hence, it is clear that a duality in terms of interpretation, analogous to that which affects probability (limiting relative frequency vs degree of belief), also affects possibility theory (degree of necessity/possibility vs lower/upper probabilities) and the theory of belief functions (degree of belief/plausibility vs lower/upper probabilities). Developing methods based on interpretations other than with reference to lower/upper probabilities represents a distinct development direction for uncertainty representation in risk analysis. Phrases such as 'degree of possibility' in possibility theory and 'degree of belief' in evidence theory do not provide sufficiently clear interpretations. This is the motivation for Cooke [64] asking for an 'operational definition' of the possibility function in possibility theory (and the membership function in fuzzy set theory). One key challenge is hence to develop or apply a clear interpretation (operational definition) of these concepts, and then to develop appropriate measurement/elicitation procedures.

\section{HYBRID REPRESENTATIONS OF UNCERTAINTY}

Probability-bound analysis (item a) in the first list in Section 1) is an example of how research has also been directed towards the combination of different representations, in this case probabilistic analysis and interval analysis. Another example is probabilistic analysis and 
possibility theory, where the uncertainties related to some of the parameters of a model are represented by probability distributions and the uncertainties related to the remaining parameters are represented by possibility distributions; see e.g. [65] and the applications in $[66,67,68]$. Probability and possibility distributions are special cases of belief functions and integrative work has also been carried out in the framework of belief functions theory; see e.g. [69,70,71]. A hybrid method has also been developed to combine nonparametric predictive inference (NPI) and the standard Bayesian framework; see [72].

The combination of uncertainty representations implies that different representations apply to different situations. Unfortunately, authoritative guidance is hard to find on when to use probability and when to use alternative representations in the context of risk assessment. The argument often seems to be that probability is the appropriate representation of uncertainty only when a sufficient amount of data exists on which to base the assignment of the probability (distribution) in question; however, it is not obvious how to make such a prescription operational [73 p. 33]: Consider the representation of uncertainty about the parameter(s) of a frequency probability model. If a sufficiently large amount of data exists, there would be no uncertainty about the parameter(s) and hence no need for a representation of such uncertainty. When is there enough data to justify probability, but not enough to accurately specify the true value of the parameter in question and thus make single-valued probability as an epistemic concept superfluous?

As an example [73 p. 33], consider the representation of uncertainty about the parameter $\mathrm{p}$ of a Bernoulli random quantity $\mathrm{X}_{1}$ for which no observations are available and for which it is considered difficult to have any well-founded opinions. A typical ignorance prior for $\mathrm{p}$ would be 
a beta probability distribution with parameters $\mathrm{x}_{0}$ and $\mathrm{n}_{0}$ both equal to 1 , which yields a uniform probability distribution on the unit interval and $\mathrm{P}\left(\mathrm{X}_{1}=1\right)=\mathrm{E}[\mathrm{p}]=\mathrm{x}_{0} /\left(\mathrm{x}_{0}+\mathrm{n}_{0}\right)=0.5$. The core of an often-made argument is then that, say, for the throw of an untested but unsuspected coin, most people would also assign $\mathrm{P}(\mathrm{Y}=1)=\mathrm{E}[\mathrm{q}]=0.5$ (resulting from a non-uniform probability distribution on q, centred around 0.5), where Y equals 1 if the outcome is 'heads' and 0 if 'tails', but perhaps have a (qualitatively) vastly different comprehension of the situation. One possible resolution of this problem is to assign say $\mathrm{P}\left(\mathrm{X}_{1}=1\right)=1 / 2$ and $\mathrm{P}(\mathrm{Y}=1)=100 / 200$, the denominator and numerator chosen to reflect the confidence in the probability assignment (Lindley, 1985); however, this involves the assignment of two numbers, just as for the assignment of lower and upper probabilities. Suppose that we observe realizations of the Bernoulli process and through Bayesian updating obtain $\mathrm{p}_{\mathrm{n}}=\mathrm{P}\left(\mathrm{X}_{\mathrm{n}+1}=1 \mid \mathrm{x}_{0}, \mathrm{x}_{1}, \ldots, \mathrm{x}_{\mathrm{n}}\right)=\left(\mathrm{x}_{0}+\mathrm{x}_{1}\right.$ $\left.+\ldots+\mathrm{x}_{\mathrm{n}}\right) /\left(\mathrm{x}_{0}+\mathrm{n}_{0}+\mathrm{n}\right)$. As $\mathrm{n}$ tends to infinity, we have, by the law of large numbers, that $\mathrm{p}_{\mathrm{n}}$ tends to the true value of $\mathrm{p}$. The questions then are:

- For which values of $\mathrm{n}$ is a probabilistic representation justified and for which values not?

- And when a probabilistic representation is not justified, what should be the criteria for selecting a particular representation format (interval analysis, imprecise probability, possibility theory, evidence theory, etc.)?

For the first question a pragmatic approach is probably required. Precise probability is an ideal case where no imprecision is involved; however, there will always be some degree of imprecision. On the other hand, because of the relative simplicity of using (calculating with) probabilities, it is desirable to use probability if the level of imprecision involved is considered negligible. Also, depending on the relevance of the available observations, different values of $n$ could be judged as sufficient in different situations. 
The second question points at an important direction of research in relation to the development of the hybrid approach, as well as for specific hybrid methods (interval analysis/probability, possibility/probability, etc.).

\section{SEMI-QUANTITATIVE APPROACHES}

The representations described so far are all quantitative. Another approach, which may be referred to as semi-quantitative, is based on a mixture of quantitative representations and qualitative methods. It may hence be considered a type of hybrid approach, integrating quantitative and qualitative representations. Examples of implementations include the approaches described by Aven [74,75,76] and Flage \& Aven [77], where in both cases standard probabilistic risk descriptions are supplemented by a qualitative assessment of uncertainty aspects not properly reflected by the quantitative descriptions.

A semi-quantitative approach implies, as the hybrid approach, a belief that probability is not perfect, but in addition it requires a belief that the full scope of uncertainty and risk cannot be transformed into a quantitative format, using probability or any other measure of uncertainty. Following this approach, when taken as a supplement to a precise probabilistic analysis, so-called "uncertainty factors" that are "hidden" in the background knowledge (see Mosleh and Bier [78] regarding elements in the background knowledge that can be uncertain and considered unknown quantities) of the subjective probabilities, are identified and assessed in a qualitative way. 
Using some simple procedures, the strength of the knowledge that supports the probabilistic analysis is categorized. For example a judgment is made on the knowledge being weak if one or more of these conditions are true [77]:

- The assumptions made represent strong simplifications.

- Data are not available, or are unreliable.

- There is lack of agreement/consensus among experts.

- The phenomena involved are not well understood; models are non-existent or known/believed to give poor predictions.

If, on the other hand, all of the following conditions are met, the knowledge is considered strong:

- The assumptions made are seen as very reasonable.

- Much reliable data are available.

- There is broad agreement/consensus among experts.

- The phenomena involved are well understood; the models used are known to give predictions with the required accuracy.

Cases in between are classified as having medium strength of knowledge. In addition there needs to be a sensitivity analysis of the importance of the uncertainty factor. If an unreasonable assumption is made but the overall result of the risk analysis is not very sensitive to deviations from this assumption, then the assumption is not as critical as if a small deviation leads to significant alterations to the overall result. 
More detailed schemes can be developed on the basis of judgments of the criticality of the assumptions made [76,77]. An assumption is critical the strength of knowledge supporting it is weak and at the same time deviations from it significantly increases the risk level as assessed in the risk analysis. The idea is to perform a crude risk assessment of potential deviations from the conditions/states defined by the assumptions. For example, if an assumption is made in a process plant risk analysis saying that a gas leak will be ignited immediately, then we may perform an assumption deviation risk assessment to consider the likelihood and effect of a delayed ignition. Let $\mathrm{X}$ denote the number of fatalities and suppose that the risk index of interest is the expected number of fatalities, EX. The base case assessment was that the time to ignition T would be zero, i.e. $\mathrm{E}[\mathrm{X} \mid \mathrm{T}=0]$, but we also consider say $\mathrm{T}$ equal to 30 seconds, i.e. $\mathrm{E}[\mathrm{X} \mid \mathrm{T}=30]$. The aim of the assessment is to assign a risk score for each deviation, which reflects risk related to the magnitude of the deviation and its implications. This "assumption deviation risk" score provides a measure of criticality or importance of the assumption. Depending on an overall judgment of these assumptions scores, a total strength of knowledge level is determined.

This criticality (importance) scoring of assumptions can be used as a guideline for where to place the focus to improve the risk assessment. The assumptions with the high criticality score should be examined to see if they can be dealt with in some way and removed from the highest importance category (for example, using the law of total probability). There will, however, always be some factors which a probability or other risk metrics cannot be made unconditional on, e.g. data and current phenomenological understanding. 


\section{OVERALL DISCUSSION}

We have ventured beyond probability to describe uncertainties in a risk assessment context. For that, we have considered alternative approaches for representing uncertainty that have been looked into, including those based on interval probability, possibility theory and evidence theory. We have made the point, strongly, that extending the framework for uncertainty analysis naturally leads to the extension of the framework for risk assessment and management. In much of the existing literature on the representation and analysis of uncertainty, risk is defined in relation to probability. For example using the well-known triplet definition of risk by Kaplan and Garrick [57], risk is equal to the triplet $\left(\mathrm{s}_{\mathrm{i}}, \mathrm{p}_{\mathrm{i}}, \mathrm{c}_{\mathrm{i}}\right)$, where $\mathrm{s}_{\mathrm{i}}$ is the $\mathrm{i}^{\prime}$ th scenario, $\mathrm{p}_{\mathrm{i}}$ is the probability of that scenario, and $c_{i}$ the consequence of the $i^{\prime}$ th scenario, $i=1,2, \ldots N$. In this view, risk captures: What can happen? How likely is that to happen? If it does happen, what are the consequences?

Kaplan [79] has generalised the above risk definition by defining risk as equal to the triplet $\left(\mathrm{s}_{\mathrm{i}}, \mathrm{l}_{\mathrm{i}}, \mathrm{c}_{\mathrm{i}}\right)$, where $\mathrm{s}_{\mathrm{i}}$ and $\mathrm{c}_{\mathrm{i}}$ are defined as above, and $\mathrm{l}_{\mathrm{i}}$ denotes the likelihood of $\mathrm{s}_{\mathrm{i}}$. However, only probabilistic formats are described for the likelihood component, namely frequency, (subjective) probability and probability of frequency. The latter refers to the use of subjective probability to express uncertainty about uncertain frequencies/frequentist probabilities.

Such a perspective is too narrow to accommodate the integration of different approaches for representing and characterising uncertainty and risk: a broader perspective is required to allow uncertainty representations other than probability. 
If risk is defined through probabilities as in the Kaplan and Garrick [57] and Kaplan [79] settings, we need to clarify what probability means. It cannot obviously be a subjective definition, as we seek a general framework that extends beyond such type of probabilities. Hence, probability must refer to a frequentist concept. However, frequentist probabilities cannot be justified in cases of non-repeatability and therefore cannot serve as a general concept for risk assessment, applicable to all types of uncertainty representations. We, consequently, have to leave the probability-based risk concepts, and extend to perspectives on risk that are based on uncertainty instead of probability.

One of the most general risk perspectives is the so-called $(C, U)$ risk perspective, where risk is understood as the two-dimensional combination of the (severity of the) consequences $\mathrm{C}$ of an activity and associated uncertainties $\mathrm{U}$ (what will $\mathrm{C}$ be?) [80,81]. This perspective is closely linked to some common risk perspectives in social sciences $[82,83,84]$, which express that risk is basically the same as consequences $\mathrm{C}$ or events that could lead to $\mathrm{C}$. The definitions of risk are different, but when it comes to the way risk is to be described there are strong similarities as the C-type perspective also covers consequences and uncertainties.

The knowledge dimension enters the scene when we try to describe or measure risk. A risk description is obtained by specifying the consequences $\mathrm{C}$ and using a description (measure) of uncertainty Q (which could be probability or any other measure - measure is here interpreted in a wide sense). Specifying the events/consequences means to identify a set of events/quantities of interest $C^{\prime}$ that characterise the events/consequences $C$. An example of $C^{\prime}$ is the number of fatalities. Depending on the principles laid down for specifying $\mathrm{C}$ and on the choice of Q, we 
obtain different perspectives on how to describe/measure risk. As a general description of risk, we can write $\left(\mathrm{C}^{\prime}, \mathrm{Q}, \mathrm{K}\right)$, where $\mathrm{K}$ is the knowledge that the specification of $\mathrm{C}^{\prime}$ and the assignment $\mathrm{Q}$ is based on. Hence, following this definition, there is a sharp distinction between the risk concept per se, and how risk is measured or described.

Instead of $(C, U)$, we often write $(A, C, U)$, when we would like to focus on hazards/threats/opportunities A. Similarly we write $\left(A^{\prime}, C^{\prime}, Q, K\right)$ in place of (C', Q, K). Vulnerability given A, can then be defined as (C,U|A), and a vulnerability description covers $\left(\mathrm{C}^{\prime}, \mathrm{Q}, \mathrm{K} \mid \mathrm{A}\right)$; i.e., vulnerability given an event $\mathrm{A}$ is basically risk conditional on this event.

We see that such a way of understanding and describing risk allows for all type of uncertainty representations, and it could consequently serve as a basis for a unified perspective for treating uncertainties in risk assessments.

\section{CONCLUSIONS}

In the present paper, we have highlighted foundational issues for the representation of uncertainty in risk assessment as well as discussed the practical implications, concerns and challenges. We have looked into five principal directions:

i) Subjective probability

ii) Non-probabilistic representations with the interpretation as lower and upper probabilities

iii) Non-probabilistic representations with interpretations other than lower and upper probabilities (degree of belief, degree of possibility etc.) 
iv) Hybrid combinations of probabilistic and non-probabilistic representations

v) Semi-quantitative approaches

These directions are not mutually exclusive, as for example (iv) could be based on a combination of (i) and (ii), and (v) could be seen as a special case of (iv), since it is based on the combination a quantitative approach (i.e. (i), (ii) or (iii)) and some qualitative assessments.

Subjective probability is currently the most common approach for treating also epistemic uncertainty in risk analysis. We have reflected on the position that "probability is perfect", on one side, and on the need for an extended framework for risk assessment that reflects the separation that practically exists between analyst and decision maker, on the other side.

We have argued that we need to see beyond probability to adequately reflect uncertainties in a risk assessment context. However, we have highlighted that how this should be done is not straightforward. A handful of approaches are out there, but they are of not easily implemented in practice. More research has to be carried out to bring these alternative approaches to an operative state where they can in fact be used in practice, when needed. The development in this direction should have the clear aim of obtaining a unified perspective (covering concepts, principles, theories and operative approaches) for the representation and characterisation of risk and uncertainty, by linking probability and alternative representations of uncertainty.

Furthermore, we support that a framework for risk assessment needs to allow for both qualitative and quantitative approaches. Earlier research has to a large extent been quantitative, but we have underlined that the full scope of the risks and uncertainties cannot be transformed into a 
mathematical formula, using probabilities or other quantitative measures of uncertainty. Numbers can be generated, but these alone would not serve the purpose of the risk assessment: to reveal and describe the risks and uncertainties. Qualitative approaches linked to probability exist (see Section 6), but similar approaches have not been developed for the alternative quantitative approaches (probability-bound analysis, imprecise probabilities, possibility theory, evidence theory).

Finally, earlier attempts at integration (hybrid probability and possibility approaches) have been based on the idea that there exists one and only one appropriate representation in a specific case (e.g. possibility representation if the information is poor and subjective probabilities if the information is strong). We believe that the variety of decision-making situations calls for a unified perspective that allows the use of several approaches for representing and characterising the risk and uncertainties. To inform the decision maker, both subjective probabilities and imprecision intervals may be used, as these approaches could capture different types of information and knowledge important for the decision maker. In addition qualitative approaches could be incorporated to provide an even more nuanced characterisation of the risk and uncertainties.

\section{ACKNOWLEDGEMENTS}

The authors are grateful to two anonymous reviewers for useful comments and suggestions to earlier versions of this article. 
For R. Flage and T. Aven the work on this article has been partly funded by the PETROMAKS2 programme of the Research Council of Norway. The financial support is gratefully acknowledged.

\section{REFERENCES}

1. Nuclear Regulatory Commission. Reactor Safety Study, an Assessment of Accident Risks. Wash 1400. Report NUREG-75/014. Washington, DC. US Nuclear Regulatory Commission, Washington, DC, 1975.

2. Rechard RP. Historical relationship between performance assessment for radioactive waste disposal and other types of risk assessment. Risk Analysis 1999; 19(5): 763-807.

3. Rechard RP. Historical background on performance assessment for the waste isolation pilot plant. Reliability Engineering and System Safety 2000; 69: 5-46.

4. Jonkman SN, van Gelder PHAJM, Vrijling, JK. An overview of quantitative risk measures for loss of life and economic damage. Journal of Hazardous Materials 2003; 99(1): 1-30.

5. Apostolakis G. The concept of probability in safety assessments of technological systems. Science 1990; 250(4986): 1359-1364.

6. Aven T. Foundations of Risk Analysis: A Knowledge and Decision-Oriented Perspective. 2nd ed. Chichester: Wiley, 2012.

7. Helton JC, Burmaster DE. Guest editorial: treatment of aleatory and epistemic uncertainty in performance assessments for complex systems. Reliability Engineering and System Safety 1996; 54: 91-94. 
8. Winkler RL. Uncertainty in probabilistic risk assessment. Reliability Engineering and System Safety 1996; 54: 127-132.

9. Apostolakis G. The concept of probability in safety assessments of technological systems. Science 1990; 250(4986): 1359-1364.

10. Blockley DI, Godfrey PS. Doing it Differently. London: Thomas Telford, 2000.

11. Blockley DI, Godfrey PS. Measuring judgements to improve performance. Civil Engineering Proceedings ICE, 2005; 158: 124-129.

12. Helton JC. Quantification of margins and uncertainties: Conceptual and computational basis. Reliability Engineering and System Safety 2011; 96: 976-1013.

13. Bedford T, Cooke RM. Probabilistic Risk Analysis: Foundations and Methods. Cambridge: Cambridge University Press, 2001.

14. Nuclear Regulatory Commission. NUREG-1855 - Guidance on the Treatment of Uncertainties Associated with PRAs in Risk-Informed Decision Making. Main Report. Vol. 1, 2009.

15. Savage, LJ. Foundations of Statistics. New York: Dover, 1972.

16. Coolen FPA. On the use of imprecise probabilities in reliability. Quality and Reliability Engineering International 2004; 20: 193-202.

17. Coolen FPA, Utkin LV. Imprecise probability: A concise overview. In: Aven T, Vinnem JE (eds). Risk, Reliability and Societal Safety. Proceedings of the European Safety and Reliability Conference (ESREL), Stavanger, Norway, 25-27 June 2007. London: Taylor \& Francis, 2007. pp. 1959-1966.

18. Utkin LV, Coolen FPA. Imprecise reliability: An introductory overview. In: Levitin G (ed.). Computational Intelligence in Reliability Engineering - New Metaheuristics, Neural and Fuzzy Techniques in Reliability. Berlin, Heidelberg: Springer, 2007. pp. 261-306. 
19. Walley P. Statistical Reasoning with Imprecise Probabilities. London: Chapman and Hall, 1991.

20. Weichselberger K. The theory of interval-probability as a unifying concept for uncertainty. International Journal of Approximate Reasoning 2000; 24: 149-170.

21. Ferson S, Hajago JG. Arithmetic with uncertain numbers: rigorous and often best possible answers. Reliability Engineering and System Safety 2004; 85: 135-152.

22. Karanki DR, Kushwaha HS, Verma AK, Ajit S. Uncertainty Analysis based on Probability Bounds (p-box) Approach in Probabilistic Safety Assessment. Risk Analysis 2009; 29(5): 662-675.

23. Unwin SD. A fuzzy set theoretic foundation for vagueness in uncertainty analysis. Risk Analysis 1986; 6(1): 27-34.

24. Zadeh LA. Fuzzy sets. Information and Control 1965; 8: 338-353.

25. Zadeh LA. Probability measures of fuzzy events. Journal of Mathematical Analysis and Applications 1968; 23: 421-427.

26. Dubois D. Possibility theory and statistical reasoning. Computational Statistics and Data Analysis 2006; 51: 47-69.

27. Dubois D, Prade H. Possibility Theory: An Approach to Computerized Processing of Uncertainty. New York: Plenum Press, 1988.

28. Zadeh LA. Fuzzy sets as a basis for a theory of possibility. Fuzzy Sets and Systems 1978; $1: 3-28$.

29. Shafer G. A Mathematical Theory of Evidence. Princeton: Princeton University Press, 1976.

30. Ross TJ, Booker JM, Parkinson WJ (eds). Fuzzy Logic and Probability Applications: Bridging the Gap. Philadelphia, PA: SIAM, 2002. 
31. Blockley DI. Analysing uncertainties: towards comparing Bayesian and interval probabilities. Mechanical Systems and Signal Processing 2012; 37: 30-42.

32. JRR. Special issue on uncertainty in engineering risk and reliability. Journal of Risk and Reliability 2010; 224(4). Coolen, F.P.A., Oberguggenberger, M. and Troffaes, M. (eds.).

33. JSTP. Special issue on imprecision in Journal of Statistical Theory and Practice 2009; 3(1). Coolen-Schrijner, P., Coolen, F.P.A., Troffaes, M. and Augustin, T. (eds).

34. RESS. Special issue on alternative representations of epistemic uncertainty in Reliability Engineering and System Safety 2004; 88(1-3). Helton, J.C. and Oberkampf, W.L. (eds.).

35. Dubois D. Representation, propagation and decision issues in risk analysis under incomplete probabilistic information. Risk Analysis 2010; 30: 361-368.

36. Aven T, Zio E. Some considerations on the treatment of uncertainties in risk assessment for practical decision-making. Reliability Engineering and System Safety 2011; 96: 6474.

37. Ferson S, Ginzburg LR. Different methods are needed to propagate ignorance and variability. Reliability Engineering and System Safety 1996; 54: 133-144.

38. Berger J. An overview of robust Bayesian analysis. Test 1994; 3: 5-124.

39. Coolen F, Augustin, CT, Troffaes MCM. Imprecise probability. In: M. Lovric (ed.) International Encyclopedia of Statistical Science 2010. pp. 645-648.

40. Klir GJ. Generalized information theory: aims, results, and open problems. Reliability Engineering and System Safety 2004; 85: 21-38.

41. Dempster AP. Upper and lower probabilities induced by a multivalued mapping. The Annals of Mathematical Statistics 1967; 38: 325-339.

42. Nguyen HT. An Introduction to Random Sets. CRC Press: Boca Raton, Florida, 2006. 
43. Dubois D, Prade H. Formal representations of uncertainty. In: Bouyssou, D, Dubois, D, Pirlot, M \& Prade, H (eds). Decision-Making Process - Concepts and Methods. London: Wiley-ISTE, 2009.

44. Walley P. Inferences from Multinomial Data: Learning about a Bag of Marbles [With discussion]. Journal of the Royal Statistical Society, Series B (Methodological) 1996; 58(1): $3-57$.

45. Cooke R. The anatomy of the squizzel: The role of operational definitions in representing uncertainty. Reliability Engineering and System Safety 2004; 85: 313-319.

46. Smets P. What is Dempster-Shafer's model? In: Yager RR, Fedrizzi M, Kacprzyk J (eds) Advances in The Dempster-Shafer Theory of Evidence, Wiley, San Mateo, CA, 1994, pp. 5-34.

47. Lindley DV. The philosophy of statistics. The Statistician 2000; 49(3): 293-337.

48. Aven T. On the interpretations of alternative uncertainty representations in a reliability and risk analysis context. Reliability Engineering and System Safety 2011; 96: 353-360.

49. Bernardo JM, Smith AFM. Bayesian Theory. Wiley, Chichester, 1994.

50. North W. Probability theory and consistent reasoning, Risk Analysis 2010; 30(3): 377380.

51. Aven T, Baraldi P, Flage R, Zio E. Uncertainty in Risk Assessment: The Representation and Treatment of Uncertainties by Probabilistic and Non-Probabilistic Methods. Wiley, Chichester, 2014.

52. Cooke RM. Deep and Shallow Uncertainty in Messaging Climate Change. In: In: Steenbergen RDJM, van Gelder PHAJM, Miraglia S \& Vrouwenvelder ACWM (eds) Safety, Reliability and Risk Analysis: Beyond the Horizon. Proceedings of the European 
Safety and Reliability Conference 2013 (ESREL 2013), Amsterdam, the Netherlands, 29 September - 2 October 2013. CRC Press.

53. Blockley DI. Fuzziness and probability: a discussion of Gaines' axioms. Civil Engineering Systems 1985; 2: 195-200.

54. Wu JS, Apostolakis GE, Okrent D. Uncertainties in system analysis: Probabilistic versus nonprobabilistic theories. Reliability Engineering \& System Safety 1990; 30: 163-181.

55. Destercke S, Dubois D, Chojnacki E. Unifying practical uncertainty representations - Part I: Generalized p-boxes. International Journal of Approximate Reasoning 2008; 49: 649663. Part II: Clouds; 49: 664-677.

56. Singpurwalla ND. Reliability and Risk: A Bayesian Perspective. Chichester: Wiley, 2006.

57. Kaplan S, Garrick BJ. On the quantitative definition of risk. Risk Analysis 1981; 1(1): 1127.

58. Lindley DV. The philosophy of statistics. The Statistician 2000; 49(3): 293-337.

59. Lindley DV. Understanding Uncertainty. Hoboken, NJ: Wiley, 2006.

60. Bernardo JM, Smith AFM. Bayesian Theory. Chichester: Wiley, 1994.

61. O'Hagan A, Oakley JE. Probability is perfect, but we can't elicit it perfectly. Reliability Engineering and System Safety 2004; 85: 239-248.

62. North DW. Probability theory and consistent reasoning. Risk Analysis 2010; 30(3): 377380.

63. Shafer G. Perspectives on the theory and practice of belief functions. International Journal of Approximate Reasoning 1990; 4: 323-362.

64. Cooke RM. The anatomy of the squizzel: The role of operational definitions in representing uncertainty. Reliability Engineering and System Safety 2004; 85: 313-319. 
65. Baudrit C, Dubois D, Guyonnet D. Joint propagation and exploitation of probabilistic and possibilistic information in risk assessment. IEEE Transactions on Fuzzy Systems 2006; 14(5): 593-608.

66. Helton JC, Johnson JD, Oberkampf WL. An exploration of alternative approaches to the representation of uncertainty in model predictions. Reliability Engineering and System Safety 2004; 85(1-3): 39-71.

67. Baraldi P, Zio E. A combined Monte Carlo and possibilistic approach to uncertainty propagation in event tree analysis. Risk Analysis 2008; 28(5): 1309-1325.

68. Flage R, Baraldi P, Aven T, Zio E. Probabilistic and possibilistic treatment of epistemic uncertainties in fault tree analysis. Risk Analysis 2013; 33(1): 121-133.

69. Dempster AP, Kong A. Uncertain evidence and artificial analysis. Journal of Statistical Planning and Inference 1988; 20(3): 355-368.

70. Almond RG. Graphical Belief Models. Chapman and Hall: London, 1995.

71. Démotier S, Schön W, Denoeux T. Risk Assessment Based on Weak Information using Belief Functions: A case study in water treatment. IEEE Trans. on Systems, Man and Cybernetics C 2006; 36: 382-396.

72. Montgomery V. New statistical methods in risk assessment by probability bounds. PhD thesis, Durham University, UK, 2009.

73. Flage R. Contributions to the treatment of uncertainty in risk assessment and management. PhD Thesis No. 100, University of Stavanger, Norway, 2010.

74. Aven T. Risk Analysis: Assessing Uncertainties beyond Expected Values and Probabilites. Chichester: John Wiley, 2008.

75. Aven T. A semi-quantitative approach to risk analysis, as an alternative to QRAs. Reliability Engineering and System Safety 2008; 93: 768-775. 
76. Aven T. On the practical implications of the new risk perspectives. Paper submitted for possible publication, 2013.

77. Flage R, Aven T. Expressing and communicating uncertainty in relation to quantitative risk analysis (QRA). Reliability \& Risk Analysis: Theory \& Applications 2009; 2(13): 918.

78. Mosleh A, Bier VM. Uncertainty about probability: a reconciliation with the subjectivist viewpoint. IEEE Transactions on Systems, Man, and Cybernetics. Part A - Systems and Humans 1996; 26(3): 303-310.

79. Kaplan S. The words of risk analysis. Risk Analysis 1997; 17: 407-417.

80. Aven T. On how to define, understand and describe risk. Reliability Engineering and System Safety 2010; 95(6): 623-631.

81. Aven T, Renn O. (2009) On risk defined as an event where the outcome is uncertain. Journal of Risk Research 12, 1-11.

82. Rosa EA. Metatheoretical foundations for post-normal risk. Journal of Risk Research 1998; 1: 15-44.

83. Rosa EA. The logical structure of the social amplification of risk framework (SARF); Metatheoretical foundations and policy implications. Pp. 47-79 in Pidgeon N, Kasperson RE, Slovic P (eds). The Social Amplification of Risk. Cambridge, UK: Cambridge University Press, 2003.

84. Renn O. Risk Governance. White paper no. 1, International Risk Governance Council, Geneva, 2005. 